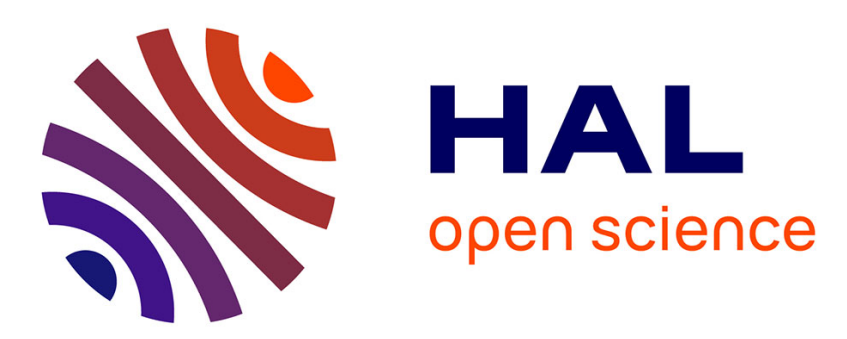

\title{
Performance measures for a deteriorating system subject to imperfect maintenance and delayed repairs
}

\author{
I. T. Castro, Sophie Mercier
}

\section{To cite this version:}

I. T. Castro, Sophie Mercier. Performance measures for a deteriorating system subject to imperfect maintenance and delayed repairs. Proceedings of the Institution of Mechanical Engineers, Part O: Journal of Risk and Reliability, 2016, 230 (4), pp.364-377. 10.1177/1748006X16641789 . hal01331121

\section{HAL Id: hal-01331121 \\ https://hal.science/hal-01331121}

Submitted on 14 Mar 2018

HAL is a multi-disciplinary open access archive for the deposit and dissemination of scientific research documents, whether they are published or not. The documents may come from teaching and research institutions in France or abroad, or from public or private research centers.
L'archive ouverte pluridisciplinaire HAL, est destinée au dépôt et à la diffusion de documents scientifiques de niveau recherche, publiés ou non, émanant des établissements d'enseignement et de recherche français ou étrangers, des laboratoires publics ou privés. 


\title{
Performance measures for a deteriorating system subject to imperfect maintenance and delayed repairs
}

\author{
I.T. Castro $^{(1)}$, Sophie Mercier ${ }^{(2)}$ \\ ${ }^{(1)}$ University of Extremadura, Department of Mathematics, \\ Escuela Politécnica, Avenida de la Universidad 10003, Cáceres, Spain \\ Email: inmatorres@unex.es \\ ${ }^{(2)}$ Université de Pau et des Pays de l'Adour, \\ Laboratoire de Mathématiques et de leurs Applications - Pau (UMR CNRS 5142), \\ Batiment IPRA, Avenue de l'Université, F-64013 PAU cedex, France \\ Email: sophie.mercier@univ-pau.fr
}

February 9, 2016

\begin{abstract}
A system subject to an accumulating deterioration and continuous monitoring is analyzed in this paper. The system deterioration is modelled using a gamma process and the system is considered as failed when its degradation level exceeds a failure threshold. The maintenance team lasts a fixed time to start the maintenance actions. To prevent downtime, an alert signal is sent in advance to the maintenance team when the degradation level of the system exceeds a preventive threshold. At the maintenance time, three maintenance actions can be performed: preventive replacement, corrective replacement and repair. We assume that the repair is imperfect in the sense that it reduces a part of the degradation accumulated by the system from the last maintenance action. Under these assumptions, integral equations fulfilled by different performance measures are obtained. Numerical examples are given that illustrate the analytical results.

Index Terms- Imperfect maintenance, gamma process, semi-Markov process, Markov renewal equation, delayed repair, maintenance policy, Monte Carlo method.
\end{abstract}

\section{Introduction}

Performance measures are used in various stages of the system life cycle to measure and predict the system behaviour. Two traditional performance measures that describe the functioning of the system are the reliability and the pointwise availability. The pointwise availability at time $t$ is defined as the probability that a system is operational at time $t$. Mercier [16] presented different stochastic models and exposed some tools for the availability assessment of a system. On the other hand, the reliability at time $t$ is defined as the probability that the system lifetime exceeds $t$. A general performance measure that generalizes the previous measures is the interval reliability defined as the probability for the system to remain operational throughout a given time interval [2]. That is, denoting by $Y_{u}$ the system state at time $u$ and by $\mathbb{U}$ the subset of operating states of a system, the interval reliability is defined as

$$
I R(t, t+x) \equiv \mathbb{P}\left(Y_{u} \in \mathbb{U}, t \leq u \leq t+x\right) .
$$


The interval reliability includes both reliability and pointwise availability as particular cases, see [5] and subsection 4.4 for details.

Markov models with finite state spaces are commonly used tools for the evaluation of transient performance measures of a system. For Markov models, the holding times are exponentially distributed which eases the calculus of the performance measures. However, the assumption of exponential holding times is often too restrictive. Considering more general holding-times leads to semi-Markov processes, which are less amenable to analyze but provide more flexible models. By the use of semi-Markov models, most transient performance measures fulfill a set of integral equations named Markov renewal equations [12]. The Markov renewal equations can be seen as a set of Volterra integral equations of the second kind. Even in the case of a finite state space, there generally exists no solution in closed form to these equations, which have to be solved numerically [15]. A first possibility is to use Laplace transform techniques, which requires numerical inversion of Laplace transforms. Due to the instability of the numerical inversion, this technique is not always an appropriate method for its numerical computation (see [4] and [7]). Another possibility is to use two-point trapezoidal rules $[5,6]$, or other discretization techniques $[9,15]$ which allow to get bounds for the performance measures of interest. Then, even for a semi-Markov process with finite state space, the computation of most transient performance measures requires the use and development of specific numerical tools for the solving of the Markov renewal equations.

In this paper, we analyze some performance measures for a continuously monitored system with gamma process deterioration and subject to a Condition-Based Maintenance (CBM) program. The setting of the problem is inspired by the framework exposed in [17]. We assume that the system fails when its degradation level exceeds a failure threshold $L$. When scheduled, the operation of the maintenance team begins after a constant delay time $\tau$. This delay time could stand for the maintenance resources mobilization (tools, maintenance crew, ...). Due to this delay and trying to avoid the system downtime, the call to the maintenance team is scheduled when the degradation level of the system exceeds a preventive threshold $M(0<M<L)$. At the arrival of the maintenance team, if the system fails during the delay time, the system is replaced by a new one (corrective replacement). Otherwise, it depends on the degradation level of the system when the maintenance team arrives: if the system is very deteriorated, it is preventively replaced; if the system is still in rather good health, it is kept going on, after some adjustments however. As an example for this kind of adjustments, one may think of the ballast compacting of a railway track, which allows to improve the so-called levelling of the track but do not allow to put the track geometry back to its initial perfect state [13]. In a more general setting, the adjustments that we have in mind lower the system deterioration level but remain imperfect, in the sense that they do not bring the system back to its brand new state. They hence correspond to some imperfect repair. The effect of the imperfect maintenance on the degradation process has been analyzed under different approaches in the literature. In [8], [13] and [18], after an imperfect maintenance, the system degradation is reduced according to a random continuous random variable. In Farahani [10], the impact of the imperfect maintenance is showed in the parameters of the gamma process that change after an imperfect maintenance action. In [17], mimicking a first-order Arithmetic Reduction of Age for recurrent events, imperfect maintenance actions reduce the age of the system.

Following the spirit of virtual age models for recurrent events [11] and analogously to the approach of [19], we assume that each imperfect maintenance action reduce the degradation level accumulated since the last maintenance action. Under this approach, the functioning of the system is described as a semi-regenerative process with continuous state space. Transient performance measures are proved to fulfill Markov renewal equations. Since the state space is continuous, the numerical resolution of the Markov renewal equation requires a high computational effort. In this paper, the performance measures are approximated not only using Monte Carlo simulation but 
also using a numerical method that combines numerical integration, interpolation techniques and Monte Carlo simulation.

The envisioned performance measures are the interval reliability, the reliability, the pointwise availability and the expected cumulated uptime. A first interest of these indicators is that they allow for a better understanding of the performance of the CBM policy. A second interest is that they could be used for maintenance optimization purpose, where level $M$ might be chosen to optimize one among these performance measures, eventually under constraints based on the other indicators. As an example, one could imagine be interested in finding $M$ which maximizes the expected cumulated uptime on a fixed foreseen operation horizon $\left[0, t_{0}\right]$ under the constraint that the poinwise availability should remain above a given availability threshold up to time $t_{0}$. One could also classically consider maximizing a cumulated cost function on $\left[0, t_{0}\right]$ constructed on the proposed indicators (which could for instance include a unitary benefit per unit time when the system is up and a unitary unavailability cost when it is down).

The paper is structured as follows. In Section 2, the framework of the functioning of the system is described. Section 3 develops some preliminary mathematical results. Section 4 is focused on the calculus of the performance measures and on the Markov renewal equations that they fulfill. Section 5 shows some numerical examples of the measures calculated previously and Section 6 concludes.

\section{Framework of the problem}

We consider a unitary system working under the following scheme.

1. The system is subject to an intrinsic deterioration modelled by a gamma process $\left\{X_{t}, t \geq 0\right\}$, where the degradation of the system at time $t, X_{t}$, is gamma distributed $\Gamma(\alpha t, \beta)$ with probability distribution function (p.d.f.)

$$
f_{t}(x)=\frac{\beta^{\alpha t}}{\Gamma(\alpha t)} x^{\alpha t-1} e^{-\beta x} \mathbf{1}_{\mathbb{R}_{+}}(x) \quad \alpha>0, \beta>0 .
$$

We recall that a gamma process is a Lévy process (a process with homogeneous and independent increments) with Lévy measure given by

$$
\mu(d s)=\alpha \frac{e^{-\beta s}}{s} \mathbf{1}_{\mathbb{R}_{+}^{*}}(s) d s,
$$

where $\mathbf{1}_{\{\}}$stands for the indicator function. Let $F_{t}\left(\bar{F}_{t}\right)$ be the cumulative distribution function (survival function) of $X_{t}$, respectively.

2. We assume that the system fails when its degradation level exceeds the failure threshold $L$. Let $\sigma_{L}$ be the time to the system failure, that is,

$$
\sigma_{L}=\inf \left(t>0: X_{t}>L\right)
$$

3. Maintenance team needs $\tau$ time units to arrive and start the repair. To avoid the system downtime, an alert signal is sent to the maintenance team as soon as the system degradation reaches a preventive threshold $M(0<M<L)$, namely at time $\sigma_{M}$, with

$$
\sigma_{M}=\inf \left(t>0: X_{t}>M\right)
$$

At time $\sigma_{M}+\tau$, maintenance tasks start under the following scheme. 
- If the system is down at the maintenance team's arrival, the system is replaced by a new one (corrective replacement).

- If the system is working at the maintenance team's arrival, the maintenance action depends on the system deterioration level:

- If the system is not too much deteriorated, an imperfect repair is performed. This repair removes a part (the $\rho$ per cent) of the deterioration accumulated by the system since the last maintenance action (or since $t=0$ ), where $\rho \in] 0,1[$. This imperfect repair is performed only if it allows to bring the system deterioration level below $M$. In that case, the system is said to be maintainable.

- If the system is too deteriorated, the system is considered to be unmaintainable and a preventive replacement is performed. This case happens when, after imperfect repair, the degradation level of the system would still exceed $M$.

All the maintenance actions are considered as instantaneous.

Let $\left\{Y_{t}, t \geq 0\right\}$ be the process that describes the evolution of the maintained system. We also set $\left\{X_{t}^{(n)}, t \geq 0\right\}$ for $n=1,2, \ldots$ to be independent copies of the gamma process $\left\{X_{t}, t \geq 0\right\}$. These copies are used to model the system intrinsic deterioration after each maintenance action at time $S_{1}, S_{2}, \ldots, S_{n}, \ldots$ Corresponding reaching times of the threshold $L$ (resp. $M$ ) are denoted by $\sigma_{L}^{(n)}$ (resp. $\sigma_{M}^{(n)}$ ) for $n=1,2, \ldots$

The first maintenance action takes place at time $S_{1}=U_{1}=\sigma_{M}^{(1)}+\tau$. We have $Y_{t}=X_{t}^{(1)}$ for all $0 \leq t<S_{1}$, and $Y_{S_{1}^{-}}\left(Y_{S_{1}}\right)$ is the system degradation level just before (after) the first maintenance action, with $Y_{S_{1}^{-}}=X_{U_{1}}^{(1)}$ (almost surely). At time $S_{1}$, different cases are possible:

- If $X_{U_{1}}^{(1)}>L$ : the system failed before $S_{1}$. An instantaneous corrective replacement takes place at time $S_{1}=\sigma_{M}^{(1)}+\tau$ and the system is replaced by a new one. Therefore: $Y_{S_{1}}=0$.

- If $X_{U_{1}}^{(1)} \leq L$ : the eventual imperfect repair would put the system back to the deterioration level $(1-\rho) X_{U_{1}}^{(1)}$.

- if $(1-\rho) X_{U_{1}}^{(1)}>M$ : the system is unmaintainable and it is preventively replaced by a new one at time $S_{1}$; hence $Y_{S_{1}}=0$.

- if $(1-\rho) X_{U_{1}}^{(1)} \leq M$ : the imperfect repair is performed and $Y_{S_{1}}=(1-\rho) X_{U_{1}}^{(1)}$.

Note that the case $X_{U_{1}}^{(1)} \leq L$ and $(1-\rho) X_{U_{1}}^{(1)}>M$ is possible only when $M<(1-\rho) L$. Also, the different cases can be summarized into:

$$
Y_{S_{1}}=\left\{\begin{array}{c}
0 \text { if } X_{U_{1}}^{(1)}>\min \left(L, \frac{M}{1-\rho}\right), \\
(1-\rho) X_{U_{1}}^{(1)} \text { otherwise. }
\end{array}\right.
$$

Starting from $Y_{S_{1}}$, the evolution of the system is assumed to be independent of $\left\{Y_{t}, t<S_{1}\right\}$ and is modelled by $\left\{X_{t}^{(2)}, t \geq 0\right\}$ up to the second maintenance action. The reaching time of level $M$ then is

$$
\inf \left(t>S_{1}: Y_{S_{1}}+X_{t-S_{1}}^{(2)}>M\right)=S_{1}+\sigma_{M-Y_{S_{1}}}^{(2)} .
$$


A second maintenance action is next planned at time $S_{2}=S_{1}+U_{2}$, with $U_{2}=\sigma_{M-Y_{S_{1}}}^{(2)}+\tau$.

More generally, assume $S_{1}, \ldots, S_{n-1}$ and $\left\{Y_{t}, t<S_{n-1}\right\}$ to be constructed, with $n \geq 2$. Let $U_{n}=\sigma_{M-Y_{S_{n-1}}}^{(n)}+\tau$ and $S_{n}=S_{n-1}+U_{n}$. We first set $Y_{t}=Y_{S_{n-1}}+X_{t-S_{n-1}}^{(n)}$ for all $S_{n-1} \leq t<S_{n}$, and consequently, the system degradation level just before the $n$-th maintenance action is $Y_{S_{n}^{-}}=$ $Y_{S_{n-1}}+X_{U_{n}}^{(n)}$ (almost surely). The degradation level just after the $n$-th maintenance action is:

$$
Y_{S_{n}}=\left\{\begin{array}{l}
0 \text { if } X_{U_{n}}^{(n)}>\min \left(L-Y_{S_{n-1}}, \frac{M-Y_{S_{n-1}}}{1-\rho}\right) \\
Y_{S_{n-1}}+(1-\rho) X_{U_{n}}^{(n)} \quad \text { otherwise. }
\end{array}\right.
$$

A new maintenance action is next planned at time $S_{n+1}=S_{n}+U_{n+1}$, with $U_{n+1}=\sigma_{M-Y_{S_{n}}}^{(n+1)}+\tau$. As in the previous maintenance times, three maintenance situations are depicted under this general setting: a corrective replacement, a preventive replacement and an imperfect repair.

After a maintenance action at time $S_{n}$, the future evolution of the maintained system $\left\{Y_{t}, t \geq S_{n}\right\}$ depends on the past $\left\{Y_{t}, t \leq S_{n}\right\}$ only through $Y_{S_{n}}$ and the process $\left\{Y_{t}, t \geq 0\right\}$ appears as a semiregenerative process with underlying Markov renewal process $\left\{\left(S_{n}, Y_{S_{n}}\right), n \in \mathbb{N}\right\}$ and inter-arrival times the $U_{n}$ 's, see [1]. The process $\left\{\left(S_{n},\left(Y_{S_{n}^{-}}, Y_{S_{n}}\right)\right), n \in \mathbb{N}\right\}$ also is a Markov renewal process, which is needed for the development of the reliability and interval reliability functions.

\section{Development of the kernels}

The aim of this section is to obtain the kernel of the Markov renewal processes of interest. To achieve this goal, we first start with the p.d.f. of $\left(S_{1}, Y_{S_{1}^{-}}\right)$, where we recall that $S_{1}$ denotes the time of the first maintenance action and $Y_{S_{1}^{-}}$the system degradation level just before the first maintenance action.

Lemma 1 The p.d.f. of $\left(S_{1}, Y_{S_{1}^{-}}\right)$is provided by:

$$
h^{M}(s, x)=\iint_{\mathbb{R}_{+}^{2}} \mathbf{1}_{\{M \leq x-y<M+u\}} f_{s-\tau}(x-y-u) f_{\tau}(y) \mu(d u) d y
$$

for all $s>\tau$ and $x>M$, and $h^{M}(s, x)=0$ elsewhere.

Proof. Based on $\left(S_{1}, Y_{S_{1}^{-}}\right)=\left(\sigma_{M}+\tau, X_{\sigma_{M}+\tau}\right)$ and on the independent increments of $\left\{X_{t}, t \geq 0\right\}$, the random vector $\left(S_{1}, Y_{S_{1}^{-}}\right)$is identically distributed as $\left(\sigma_{M}, X_{\sigma_{M}}\right)+\left(\tau, X_{\tau}^{(1)}\right)$, where $\left(\tau, X_{\tau}^{(1)}\right)$ is independent on $\left(\sigma_{M}, X_{\sigma_{M}}\right)$. The p.d.f. of $\left(S_{1}, Y_{S_{1}^{-}}\right)$hence is the convolution of the p.d.f. of $\left(\sigma_{M}, X_{\sigma_{M}}\right)$ and of the distribution of $\left(\tau, X_{\tau}^{(1)}\right)$. By Bertoin [3], the p.d.f. of $\left(\sigma_{M}, X_{\sigma_{M}}\right)$ is given by

$$
g^{M}(t, y)=\mathbf{1}_{\{M \leq y\}} \int_{0}^{\infty} \mathbf{1}_{\{y<M+s\}} f_{t}(y-s) \mu(d s),
$$

where $\mu(d s)$ denotes the Lévy measure of the gamma process $\left\{X_{t}, t \geq 0\right\}$ provided by (1). Hence

$$
h^{M}(s, x)=\int_{\mathbb{R}^{+}} g^{M}(s-\tau, x-y) f_{\tau}(y) d y=\iint_{\mathbb{R}_{+}^{2}} \mathbf{1}_{\{M \leq x-y<M+u\}} f_{s-\tau}(x-y-u) f_{\tau}(y) \mu(d u) d y .
$$


We now derive the kernel $Q_{x}(d s, d y, d z)$ of the Markov renewal process

$$
\left\{\left(S_{n},\left(Y_{S_{n}^{-}}, Y_{S_{n}}\right)\right), n \in \mathbb{N}\right\} .
$$

Setting $\mathbb{P}_{x}$ to be the conditional probability given that the system started from state $x$ :

$$
\mathbb{P}_{x}(\cdot)=\mathbb{P}\left(\cdot \mid Y_{0}=x\right),
$$

we recall that

$$
Q_{x}(d s, d y, d z)=\mathbb{P}_{x}\left(S_{1} \in d s, Y_{S_{1}^{-}} \in d y, Y_{S_{1}} \in d z\right)
$$

stands for the conditional distribution of $\left(S_{1},\left(Y_{S_{1}^{-}}, Y_{S_{1}}\right)\right)$ given that the system started from $Y_{0}=x$, which coincides with the conditional distribution of $\left(S_{n+1},\left(Y_{S_{n+1}^{-}}, Y_{S_{n+1}}\right)\right)$ given that $Y_{S_{n}}=x$ :

$$
Q_{x}(d s, d y, d z)=\mathbb{P}\left(S_{n+1} \in d s, Y_{S_{n+1}^{-}} \in d y, Y_{S_{n+1}} \in d z \mid Y_{S_{n}}=x\right) \text { for all } n \in \mathbb{N} .
$$

The proof of the following result is postponed to the Appendix.

Theorem 2 The kernel $Q_{x}(d s, d y, d z)$ of the Markov renewal process $\left\{\left(S_{n},\left(Y_{S_{n}^{-}}, Y_{S_{n}}\right)\right), n \in \mathbb{N}\right\}$ is provided by

$$
\begin{aligned}
Q_{x}(d s, d y, d z) & =\bar{q}_{x}(s, z) d s \delta_{x+\frac{z-x}{1-\rho}}(d y) d z \\
& +\mathbf{1}_{\left\{y-x>\min \left(L-x, \frac{M-x}{1-\rho}\right)\right\}} \mathbf{1}_{\{s>\tau\}} h^{M-x}(s, y-x) d s d y \delta_{0}(d z)
\end{aligned}
$$

for all $x \in[0, M]$, where

$$
\bar{q}_{x}(s, z)=\mathbf{1}_{\{0<z-x \leq \min ((1-\rho)(L-x), M-x)\}} \mathbf{1}_{\{s>\tau\}} \frac{1}{1-\rho} h^{M-x}\left(s, \frac{z-x}{1-\rho}\right)
$$

and where $\delta_{a}(d z)$ stands for the Dirac mass at point a.

The first term of the kernel corresponds to an imperfect maintenance action and the second one to a corrective or preventive replacement, where the level is set back to 0 .

We next derive the kernel $q_{x}(d s, d z)$ of the Markov renewal process $\left\{\left(S_{n}, Y_{S_{n}}\right), n \in \mathbb{N}\right\}$.

Corollary 3 The kernel $q_{x}(d s, d z)$ of the Markov renewal process $\left\{\left(S_{n}, Y_{S_{n}}\right), n \in \mathbb{N}\right\}$ is given by:

$$
q_{x}(d s, d z)=\bar{q}_{x}(s, z) d s d z+\stackrel{\circ}{q}_{x}(s) d s \delta_{0}(d z)
$$

for all $x \in[0, M]$, where

$$
\stackrel{\circ}{q}_{x}(s)=\mathbf{1}_{\{s>\tau\}} \int_{\min \left(L-x, \frac{M-x}{1-\rho}\right)}^{+\infty} h^{M-x}(s, u) d u .
$$


Proof. The kernel $q_{x}(d s, d z)$ is obtained by integrating $Q_{x}(d s, d y, d z)$ with respect of $d y$. This provides:

$$
\begin{aligned}
q_{x}(d s, d z) & =\bar{q}_{x}(s, z) d s\left(\int_{0}^{+\infty} \delta_{x+\frac{z-x}{1-\rho}}(d y)\right) d z \\
& +\mathbf{1}_{\{s>\tau\}}\left(\int_{x+\min \left(L-x, \frac{M-x}{1-\rho}\right)}^{+\infty} h^{M-x}(s, y-x) d y\right) d s \delta_{0}(d z)
\end{aligned}
$$

and the result, noticing that $\int_{0}^{+\infty} \delta_{x+\frac{z-x}{1-\rho}}(d y)=1$ and setting $u=y-x$ in the last integral.

We finally give the sub-kernel $q_{x}^{*}(d s, d z)$ of the Markov renewal process $\left\{\left(S_{n}, Y_{S_{n}}\right), n \in \mathbb{N}\right\}$ restricted to the operating states, with:

$$
q_{x}^{*}(d s, d z)=\mathbb{P}_{x}\left(S_{1} \in d s, Y_{S_{1}^{-}}<L, Y_{S_{1}} \in d z\right)
$$

(and total mass $q_{x}^{*}([\tau,+\infty[\times[0, M])$ smaller than 1$)$. The proof is postponed to the Appendix.

Corollary 4 The sub-kernel $q_{x}^{*}(d s, d z)$ is provided by:

$$
q_{x}^{*}(d s, d z)=\bar{q}_{x}(s, z) d s d z+\tilde{q}_{x}(s) d s \delta_{0}(d z)
$$

for all $x \in[0, M]$, with

$$
\tilde{q}_{x}(s)=\mathbf{1}_{\left\{x \geq \frac{M-(1-\rho) L}{\rho}\right\}}\left(\int_{\frac{M-x}{1-\rho}}^{L-x} h^{M-x}(s, u) d u\right),
$$

all $x \in[0, M]$.

The terms corresponding to the imperfect maintenance action $\left(\bar{q}_{x}(s, z) d s d z\right)$ are identical for both kernels $q_{x}(d s, d z)$ and $q_{x}^{*}(d s, d z)$. This is natural because a necessary condition for an imperfect maintenance action to be performed is that the system is not failed at the maintenance time. For this term, both unrestricted and restricted kernels to the operating states consequently coincide.

Remark 5 The system is renewed at each corrective and preventive replacement. This means that, apart from its semi-regenerative structure, the process $\left\{Y_{t}, t \geq 0\right\}$ can also be seen as a regenerative process, with replacement times as renewal times. The cycles between two renewal times however are much more difficult to analyze than the present semi-Markov cycles delimited by two maintenance actions, whatever they are. The regenerative structure of $\left\{Y_{t}, t \geq 0\right\}$ consequently does not bring much to the finite time analysis of the system. However, based on the fact that the mean hitting time $\mathbb{E}\left(\sigma_{L}\right)$ is finite for a gamma process, it is clear that the mean length of a cycle (between two replacements) is finite too, so that general theorems for regenerative processes with finite expected cycle lengths can be applied. This shows that $\left\{Y_{t}, t \geq 0\right\}$ admits a limiting distribution when $t \rightarrow \infty$, which is the mean value of $\left\{Y_{t}, t \geq 0\right\}$ on a cycle [1, Chapter VI: Thm 1.2]. Do to the complexity of the present model, this asymptotic distribution cannot however be computed explicitly.

\section{Performance measures}

In this section, we show some performance measures of the system. 


\subsection{Pointwise availability}

Given $Y_{0}=x$, let $A_{x}(t)$ be the pointwise availability of the maintained system at time $t$

$$
A_{x}(t)=\mathbb{P}_{x}\left(Y_{t}<L\right) .
$$

As $S_{1}=\tau+\sigma_{M-x}>\tau$, let us first remark that, if $t \leq \tau$, then $t<S_{1}$ and there is no maintenance action on $[0, t]$. In that case, $Y_{u}=X_{u}$ on $[0, t]$ and we simply get:

$$
A_{x}(t)=\mathbb{P}\left(\sigma_{L-x}>t\right)=\mathbb{P}\left(X_{t}<L-x\right)=F_{t}(L-x),
$$

for all $t \leq \tau$. We next envision the case where $t>\tau$.

Theorem 6 The pointwise availability fulfills the following Markov renewal equation:

$$
A_{x}(t)=G_{x}(t)+\int_{\tau}^{t} \int_{0}^{M} A_{z}(t-s) \bar{q}_{x}(s, z) d s d z+\int_{\tau}^{t} A_{0}(t-s) \stackrel{\circ}{q}_{x}(s) d s
$$

for all $t>\tau$, all $x \in[0, M]$ with

$$
G_{x}(t)=\int_{0}^{M-x} f_{t-\tau}(y) F_{\tau}(L-x-y) d y,
$$

where $\bar{q}_{x}(s, z), q_{x}(d s, d y)$ and $\stackrel{\circ}{x}_{x}(s)$ are defined in $(2-4)$.

Though a similar result is proved in [17] (with a different kernel however), we here provide a few elements of proof, for a better comprehension of the results. The interested reader may find more details in [17].

Elements of proof. Let $t>\tau$. We have:

$$
A_{x}(t)=\mathbb{P}_{x}\left(Y_{t}<L, S_{1}>t\right)+\mathbb{P}_{x}\left(Y_{t}<L, S_{1} \leq t\right)
$$

with

$$
\begin{aligned}
\mathbb{P}_{x}\left(Y_{t}<L, S_{1}>t\right) & =\mathbb{P}_{x}\left(X_{t}<L, \tau+\sigma_{M}>t\right) \\
& =\mathbb{P}\left(X_{t}<L-x, \sigma_{M-x}>t-\tau\right) \\
& =\mathbb{P}\left(X_{t}<L-x, X_{t-\tau}<M-x\right)
\end{aligned}
$$

Based on the homogeneous and independent increments of $\left(X_{t}\right)_{t \geq 0}$, the random variables $\left(X_{t}-X_{t-\tau}\right)$ and $X_{t-\tau}$ are independent with respective p.d.f.s $f_{\tau}$ and $f_{t-\tau}$. Writing $X_{t}=X_{t-\tau}+\left(X_{t}-X_{t-\tau}\right)$ and starting again from (9), we get:

$$
\begin{aligned}
\mathbb{P}_{x}\left(Y_{t}<L, S_{1}>t\right) & =\iint_{\mathbb{R}_{+}^{2}} \mathbf{1}_{\{y+z<L-x, y<M-x\}} f_{t-\tau}(y) f_{\tau}(z) d y d z \\
& =\int_{0}^{M-x} f_{t-\tau}(y) F_{\tau}(L-x-y) d y \\
& =G_{x}(t) .
\end{aligned}
$$

The second term in the right side of (8) is next dealt by conditioning with respect of the past of the process up to time $S_{1}$ and by using the Markov property at time $S_{1}$. This provides the two last terms in (6).

For the implementation of the numerical techniques developed in Section 5, we use a recursive scheme fulfilled by the pointwise availability, as provided by the following corollary (with proof postponed to the Appendix). 


\section{Corollary 7 Setting}

$$
A_{x}^{(i)}(t)=A_{x}(t)
$$

for all $(i-1) \tau<t \leq i \tau, x \in[0, M], i \geq 1$, we have:

$$
A_{x}^{(1)}(t)=F_{t}(L-x), \text { for all } 0<t \leq \tau \text {, all } x \in[0, M]
$$

and

$$
\begin{aligned}
A_{x}^{(i+1)}(t) & =G_{x}(t)+\sum_{k=1}^{i} \int_{0}^{\tau} \int_{0}^{M} A_{z}^{(k)}(k \tau-v) \bar{q}_{x}(t+v-k \tau, z) d v d z \\
& +\sum_{k=1}^{i} \int_{0}^{\tau} A_{0}^{(k)}(k \tau-v) \stackrel{\circ}{x}_{x}(t+v-k \tau) d v
\end{aligned}
$$

for all $i \tau<t \leq(i+1) \tau$, all $x \in[0, M]$, all $i \geq 1$.

\subsection{Expected cumulated uptime}

Given $Y_{0}=x$, let $m_{x}(t)$ be the expected cumulated uptime in $(0, t]$

$$
m_{x}(t)=\int_{0}^{t} A_{x}(s) d s .
$$

Let us first remark that, if $t \leq \tau$, using (5), we have

$$
m_{x}(t)=\int_{0}^{t} F_{s}(L-x) d s .
$$

For $t>\tau$, we have the following result.

Theorem 8 For $t>\tau$, the expected cumulated uptime at time $t$ fulfills the following Markov renewal equation

$$
m_{x}(t)=H_{x, \tau}(t)+\int_{0}^{M} \int_{\tau}^{t} m_{y}(t-s) q_{x}(d s, d y)
$$

where

$$
H_{x, \tau}(t)=\int_{0}^{\tau} F_{s}(L-x) d s+\int_{\tau}^{t} G_{x}(s) d s,
$$

$G_{x}$ is given by (7) and $q_{x}(d s, d y)$ denotes the kernel of the Markov renewal process $\left\{\left(S_{n}, Y_{S_{n}}\right), n \in \mathbb{N}\right\}$ given by (3).

Proof. For $t>\tau$,

$$
m_{x}(t)=\int_{0}^{\tau} A_{x}(s) d s+\int_{\tau}^{t} A_{x}(s) d s
$$

From (5) and Theorem 6, we have

$$
\begin{aligned}
m_{x}(t) & =\int_{0}^{\tau} F_{s}(L-x) d s+\int_{\tau}^{t} G_{x}(s) d s+\int_{\tau}^{t}\left(\int_{\tau}^{s} \int_{0}^{M} A_{y}(s-u) q_{x}(d u, d y)\right) d s \\
& =H_{x, \tau}(t)+\int_{0}^{M} \int_{\tau}^{t} m_{y}(t-u) q_{x}(d u, d y) .
\end{aligned}
$$

Equation (13) allows to write a recursive scheme for the computation of $m_{x}(t)$ in the same way as the one provided by Corollary 7 for the pointwise availability. 


\subsection{Reliability}

Given $Y_{0}=x$, let $R_{x}(t)$ be the reliability function of the maintained system at time $t$,

$$
R_{x}(t)=\mathbb{P}_{x}(T>t)
$$

where $T$ is the failure time of the maintained system and $t \in \mathbb{R}_{+}$. For $t \leq \tau$, the reliability is $R_{x}(t)=A_{x}(t)$. For $t>\tau$, we have the following result.

Theorem 9 For $t>\tau$, the reliability function fulfills the following Markov renewal equation

$$
R_{x}(t)=G_{x}(t)+\int_{\tau}^{t} \int_{0}^{M} R_{y}(t-s) q_{x}^{*}(d s, d y)
$$

for all $x \in[0, M]$, where $G_{x}(t)$ is given by (7) and where $q_{x}^{*}$ denotes the sub-kernel of the Markov renewal process $\left\{\left(S_{n}, Y_{S_{n}}\right), n \in \mathbb{N}\right\}$ restricted to the operating states, provided by Corollary 4 .

A proof of an equivalent result is given in [17]. Also, note that using similar methods as in e.g. [14] (with tools from [1]), one might expect to have an asymptotically exponential decay for $R_{x}(t)$ when $t \rightarrow \infty$. The study of such a property seems however rather technical and beyond the scope of the present paper.

Here again, Equation (14) allows to write a recursive scheme for the computation of $R_{x}(t)$.

\subsection{Interval reliability}

Given $Y_{0}=x$, the interval reliability $I R_{x}(t, t+s)$ is the conditional probability that the system is in the set of up states throughout the whole interval $[t, t+s](t \geq 0, s \geq 0)$

$$
I R_{x}(t, t+s)=\mathbb{P}_{x}\left(Y_{u}<L ; \forall u \in[t, t+s]\right) .
$$

For $t+s \leq \tau$, we get that $t+s<S_{1}$, hence

$$
I R_{x}(t, t+s)=\mathbb{P}_{x}\left(X_{t+s}<L\right)=F_{t+s}(L-x) .
$$

For $t+s>\tau$, the following result is obtained. The proof is postponed to the Appendix.

Theorem 10 In the case $\tau \leq t$, the interval reliability in $[t, t+s]$ fulfills the following Markov renewal equation

$$
\begin{aligned}
I R_{x}(t, t+s) & =G_{x}(t+s)+\int_{t}^{t+s} \int_{0}^{M} R_{y}(t+s-u) q_{x}^{*}(d u, d y) \\
& +\int_{\tau}^{t} \int_{0}^{M} I R_{y}(t-u, t+s-u) q_{x}(d u, d y)
\end{aligned}
$$

where $G_{x}$ is given by (7), $q_{x}^{*}$ and $q_{x}$ denote the kernel and sub-kernel given in Corollaries 3 and 4 , and $R_{y}(\cdot)$ is the reliability of the system given in Theorem 9.

In the case $t<\tau<t+s$, we get

$$
I R_{x}(t, t+s)=G_{x}(t+s)+\int_{\tau}^{t+s} \int_{0}^{M} R_{y}(t+s-u) q_{x}^{*}(d u, d y) .
$$

Here again, Equation (15) allows to write a recursive scheme for the computation of $I R_{x}(t, t+s)$. 


\section{$5 \quad$ Numerical examples}

To illustrate the analytical results, some numerical examples are showed in this section. Firstly, we shall give some examples of the performance measures computed using Monte Carlo simulation.

A system is subject to a continuous deterioration modelled by a gamma process with parameters $\alpha=1.5$ and $\beta=3$. The system fails when its degradation exceeds the failure threshold $L=9$ with expected time $\mathbb{E}\left[\sigma_{L}\right] \simeq 18.3469$ time units. A signal is sent to the maintenance team when the system degradation exceeds $M=5$ with expected time $\mathbb{E}\left[\sigma_{M}\right] \simeq 10.3728$ time units. The repair efficiency is given by $\rho=0.5$ and the delay time for the repair is $\tau=8$ time units.

Figure 1 shows the pointwise availability $A_{0}(t)$ versus $t$ given $Y_{0}=0$. The data have been obtained using Monte Carlo simulation for 21 equally spaced values from 10 to 30 with 40000 realizations in each point.

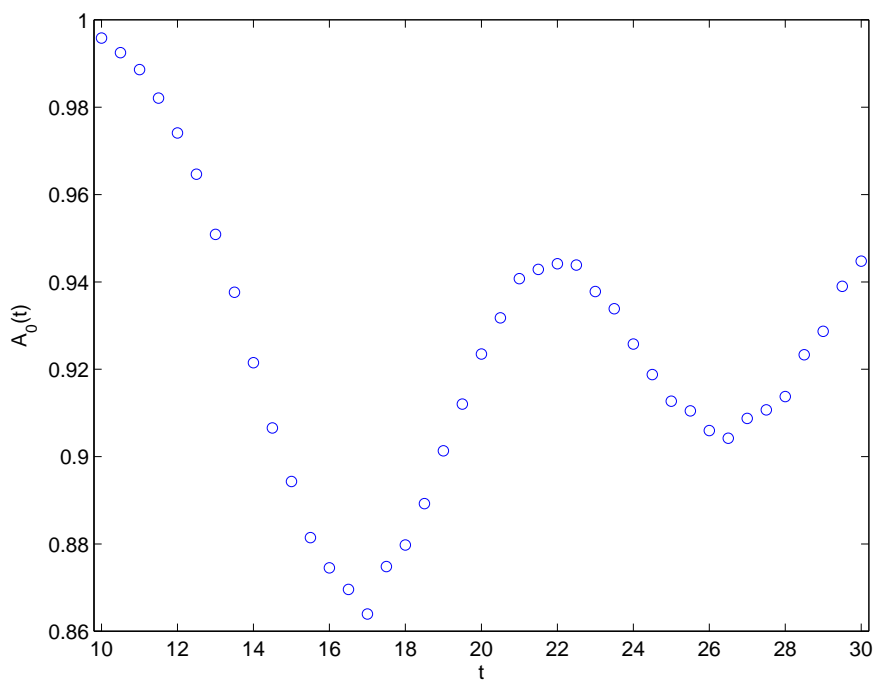

Figure 1: Pointwise availability $A_{0}(t)$ versus $t$

Based on Figure 1, the pointwise availability reaches its minimum at $t^{*} \simeq 17$ with $A_{0}(17) \simeq$ 0.8639 . It means that, for values of $t$ ranging from 10 to 30 , the probability that the system is functioning is greater than 0.8639. Also, oscillations can be observed in Figure 1. A possible explanation is the following: At the beginning, no maintenance actions are performed and the availability first decreases, due to failures. A first preventive maintenance action takes place at time $\sigma_{M}+\tau$ with mean about 18.4 time units. The impact of this action can be observed in Figure 1 , where the availability increases roughly from time 17 up to time 22 . After a while, this first preventive maintenance action has no more impact and the availability starts again to decrease. However, the time to the second preventive maintenance action is shorter than for the first one, because the first preventive maintenance action is not perfect, so that the system reaches level $M$ more quickly. This leads to some damped oscillations that can be seen in Figure 1.

Given $Y_{0}=0$, Figure 2 shows the interval reliability for different values of the left point of the interval and constant length, 10 time units, $I R_{0}(t, t+10)$. The data have been obtained using Monte Carlo simulation for 121 values of $t$ ranging from 10 to 70, with 40000 realizations in each point. Based on Figure 2, we can see that the interval reliability reaches its minimum at $t^{*} \simeq 15$ with $I R_{0}(15,25) \simeq 0.4391$. As expected from Remark 5 , we also notice the asymptotic behavior of the interval reliability, which stabilizes and tends to 0.57 when $t \rightarrow \infty$. 


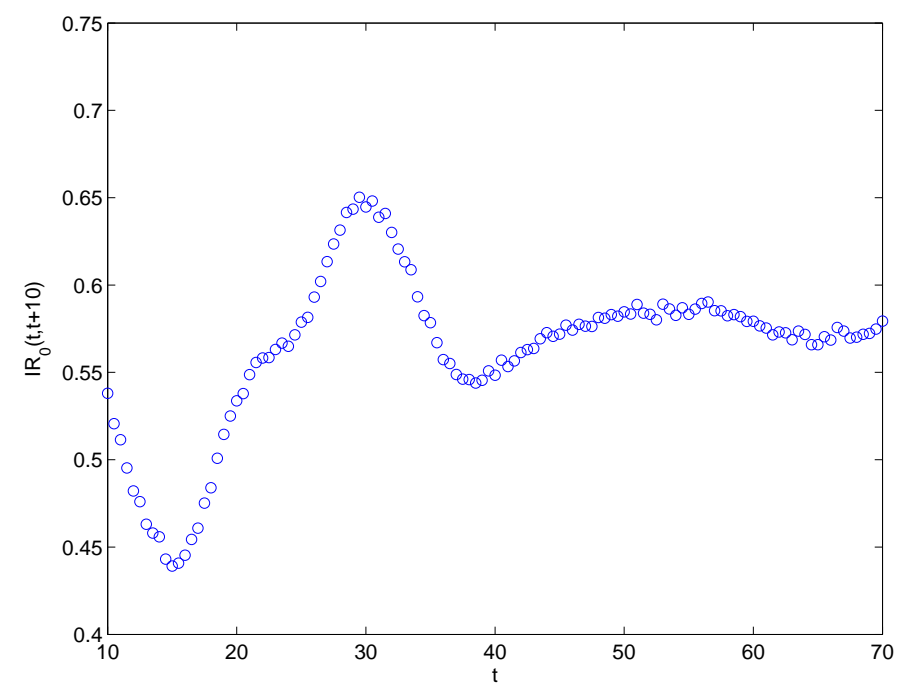

Figure 2: Interval reliability $I R(t, t+10)$ versus $t$

Using the same data set, for fixed $t=20$, Figure 3 shows the interval reliability for different values of $s$ divided by $s, \operatorname{IR}(5,5+s) / s$ given $Y_{0}=0$. The results have been obtained using Monte Carlo simulation for 40 values of $s$ ranging from 10 to 30 and 40000 simulations in each point. We see that $\operatorname{IR}(5,5+s) / s$ is a non-increasing function in $s$



Figure 3: Interval reliability $I R_{0}(5,5+s)$ versus $s$

Given $Y_{0}=0$, Figure 4 shows the mean proportion of the time that the system is working in the interval $[0, t]$, that is, $m_{0}(t) / t$ for different values of $t$. These values have been calculated using Monte Carlo simulation for 31 values of $t$ ranging from 30 to 60 with 50000 simulations in each point.

Using the same data set, that is, $L=9, M=5, \alpha=1.5, \beta=3, \tau=8, \rho=0.5$, we evaluate the system reliability. Figure 5 shows the reliability of the system at time $t$ (that is, 




Figure 4: Fraction of time that the system is up in $[0, t]$ versus $t$

$R(t)=P(T>t))$ where $T$ denotes the system lifetime. These values have been calculated using Monte Carlo simulation for 51 values from 0 to 100 with 40000 simulations in each point.

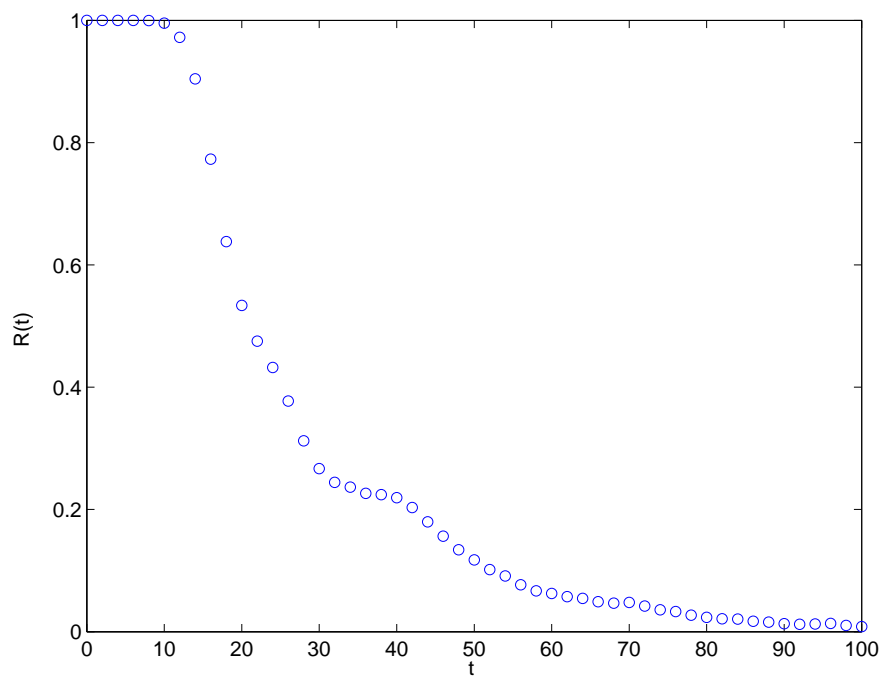

Figure 5: Probability that the system lifetime exceeds $t$ given $Y_{0}=0$

We now compute a numerical approximation of the pointwise availability, using the recursive scheme provided in Corollary 7. To begin with, we need to compute an approximation of the kernel $q_{x}(d s, d z)$. A first possibility might be to use the theoretical expression of $q_{x}(d s, d z)$ given in Corollary 3. However, the heavy tail around 0 of the Lévy measure $\mu(d x)$ of the gamma process $\left\{X_{t}, t \geq 0\right\}$ entails some numerical instability for the computation of the function $h^{M}$ (defined in Lemma 1) and hence, for the computation of $q_{x}(d s, d z)$. We consequently prefer use Monte-Carlo simulations plus interpolation techniques based on spline functions to compute an approximation 
of the kernel

$$
q_{x}(d s, d z)=\bar{q}_{x}(s, z) d s d z+\stackrel{\circ}{q}_{x}(s) d s \delta_{0}(d z) .
$$

More specifically, we compute approximations $\widehat{\bar{q}}_{x}(s, z)$ and $\widehat{\stackrel{q}{q}}_{x}(s)$ of the two functions $\bar{q}_{x}(s, z)$ and $\stackrel{\circ}{q}_{x}(s)$ involved in the kernel $q_{x}(d s, d z)$. This is done through the following steps.

1. Taking $M=5$, we first discretize the set $[0, M]$ into 50 equally spaced points from 0 to $M=5$. The corresponding grid is denoted by $E$ in the following.

2. For each fixed $x$ element of $E$, we obtain $m=480000$ simulation results for $\left(S_{1}, Y_{S_{1}}\right)$ given $Y_{0}=x$ by the Monte Carlo method. We denote by $\left(s_{i}^{x}, z_{i}^{x}\right)$ for $i=1,2, \ldots, 480000$ the results of these simulations.

3. For each fixed $x$ element of $E$, let $I_{1}^{x}=\left(\tau, \max \left(s_{i}^{x}\right)\right]$ and $I_{2}^{x}=[0, M]$ be the range of the values $\left(s_{i}^{x}, z_{i}^{x}\right)$, that is, $\left(s_{i}^{x}, z_{i}^{x}\right) \in I_{1}^{x} \times I_{2}^{x}$. We divide the intervals $I_{1}^{x}$ and $I_{2}^{x}$ into $N=299$ disjoint intervals

$$
I_{1}^{x}=I_{1,1}^{x} \cup I_{1,2}^{x} \cup I_{1,3}^{x} \cup \ldots I_{1, N}^{x}
$$

where $I_{1, i}^{x}=\left(a_{1, i}^{x}, a_{1, i+1}^{x}\right]$ for $i=1,2, \ldots, N$ and

$$
a_{1, i}^{x}=\tau+\Delta s^{x}(i-1), \quad i=1,2, \ldots, N+1
$$

and $\Delta s^{x}=\left(\max \left(s_{i}^{x}\right)-\tau\right) / N$.

In the same way

$$
I_{2}^{x}=I_{2,1}^{x} \cup I_{2,2}^{x} \cup I_{2,3}^{x} \cup \ldots I_{2, N}^{x}
$$

where $I_{2,1}^{x}=\{0\}, I_{2, i}^{x}=\left(a_{2, i}^{x}, a_{2, i+1}^{x}\right]$ for $i=2,3, \ldots N$ with

$$
a_{2, i}^{x}=\Delta z^{x}(i-2), \quad i=2, \ldots, N+1,
$$

where $\Delta z^{x}=M /(N-1)$.

For each simulated pair $\left(s_{i}^{x}, z_{i}^{x}\right)$, we find subindices $j$ and $k$ such as

$$
\left(s_{i}^{x}, z_{i}^{x}\right) \in I_{1, j}^{x} \times I_{2, k}^{x} .
$$

4. For each fixed $x$ element of $E$, we compute the function $\widehat{\bar{q}}_{x}$ (resp. $\widehat{\stackrel{q}{q}}_{x}$ ) on the grid

$$
\left\{\left(a_{1, j}, a_{2, k}\right) ; j=1,2, \ldots, N ; k=2,3, \ldots N\right\}
$$

(resp. $\left.\left\{\left(a_{1, j}\right) ; j=1,2, \ldots, N\right\}\right)$ through

$$
\begin{aligned}
& \widehat{\bar{q}}_{x}\left(a_{1, j}, a_{2, k}\right)=\frac{\sharp\left\{\left(s_{i}^{x}, z_{i}^{x}\right) \in I_{1, j}^{x} \times I_{2, k}^{x}\right\}}{m \times \Delta s^{x} \times \Delta z^{x}}, \\
& \widehat{\stackrel{q}{q}}_{x}\left(a_{1, j}\right)=\frac{\sharp\left\{\left(s_{i}^{x}, 0\right) \in I_{1, j}^{x} \times I_{2,1}^{x}\right\}}{m \times \Delta z^{x}} .
\end{aligned}
$$

5. The approximation $\widehat{\bar{q}}_{x}(s, z)\left(\right.$ resp. $\left.\widehat{\stackrel{q}{q}}_{x}(s)\right)$ is next constructed on $I_{1}^{x} \times I_{2}^{x}=\left(\tau, \max \left(s_{i}^{x}\right)\right] \times[0, M]$ (resp. $\left.I_{1}^{x}\right)$ for each $x \in[0, M]$ through the following. 
(a) If $x$ is element of $E$, the functions $\widehat{\bar{q}}_{x}(s, z)$ and $\widehat{\stackrel{q}{q}}_{x}(s)$ are already known on the grids from point 4. The two functions are extended to $I_{1}^{x} \times I_{2}^{x}$ and $I_{1}^{x}$, respectively, using interpolation techniques based on spline functions and starting from their known values on the grids. This interpolation is performed using functions interp1 and interp2 of Matlab programme for the functions $\widehat{\grave{q}}_{x}(s)$ and $\widehat{\bar{q}}_{x}(s, z)$ respectively replacing the values outside of the intervals $I_{1}^{x}$ and $I_{1}^{x} \times I_{2}^{x}$ with 0 respectively.

(b) If $x \in[0, M]$ does not belong to $E$, we search for the element $x^{*}$ of $E$ which is the nearest from $x$ and we set

$$
\widehat{\bar{q}}_{x}(s, z)=\widehat{\bar{q}}_{x^{*}}(s, z), \quad \widehat{\stackrel{q}{q}}_{x}(s)=\widehat{\grave{q}}_{x^{*}}(s),
$$

for the grid points. From (a), functions $\widehat{\bar{q}}_{x^{*}}$ and $\widehat{\stackrel{q}{q}}_{x^{*}}$ are extended to $I_{1}^{x} \times I_{2}^{x}$ and $I_{1}^{x}$.

We next use the approximated kernel to compute the availability and we consider the following procedure. We here assume that the system starts from an element $x$ of the grid $E$. If it is not the case, we just substitute $x$ by $x^{*} \in E$, as previously explained in point $\mathbf{5}$.

1. We consider grids $\left\{z_{i}, i=1,2, \ldots, n\right\},\left\{y_{i}, i=1,2, \ldots, n\right\}$ and $\left\{s_{i}, i=1,2, \ldots, n\right\}$ composed of $n=20$ equally spaced points of the intervals $[0, M-x],[0, M]$ and $[0, \tau]$ given by

$$
\begin{aligned}
z_{i} & =(i-1) \frac{M-x}{n-1}, \quad i=1,2, \ldots, n, \quad \Delta z=z_{i+1}-z_{i}, \\
y_{i} & =(i-1) \frac{M}{n-1}, \quad i=1,2, \ldots, n \quad \Delta y=y_{i+1}-y_{i} \\
s_{i} & =(i-1) \frac{\tau}{n-1}, \quad i=1,2, \ldots, n \quad \Delta s=s_{i+1}-s_{i} .
\end{aligned}
$$

We also set $z_{i}^{*}, y_{i}^{*}$ and $s_{i}^{*}$ to be the middle points of these grids, that is

$$
z_{i}^{*}=z_{i}+\Delta z / 2, y_{i}^{*}=y_{i}+\Delta y / 2, s_{i}^{*}=s_{i}+\Delta s / 2 .
$$

2. Based on the recursive scheme provided by Corollary 7 and using middle point method for approximating the integral, we estimate the pointwise availability $\widehat{A}_{x}(t)$ at time $t$ starting from $Y_{0}=x$ as follows.

- For $t<\tau$,

$$
\widehat{A}_{x}^{(1)}(t)=F_{t}(L-x)
$$

- For $i \tau<t \leq(i+1) \tau$

$$
\begin{aligned}
& \widehat{A}_{x}^{(i+1)}(t)=\sum_{j=1}^{n} f_{t-\tau}\left(z_{j}^{*}\right) F_{\tau}\left(L-x-z_{j}^{*}\right) \Delta z \\
& +\sum_{k=1}^{i} \sum_{j=1}^{n} \sum_{i=1}^{n} \widehat{A}_{y_{i}^{*}}^{(k)}\left(k \tau-s_{j}^{*}\right) \widehat{\bar{q}}_{x}\left(s_{j}^{*}+t-k \tau, y_{i}^{*}\right) \Delta y \Delta s \\
& +\sum_{k=1}^{i} \sum_{j=1}^{n} \widehat{A}_{0}^{(k)}\left(k \tau-s_{j}^{*}\right) \widehat{\grave{q}}_{x}\left(s_{j}^{*}+t-k \tau\right) \Delta s
\end{aligned}
$$




\begin{tabular}{c||c|c|c|c|c|c|c|c}
$\mathrm{t}$ & 11 & 12 & 13 & 14 & 15 & 16 & 17 & \\
\hline MC Simulation & 0.9973 & 0.9926 & 0.9831 & 0.9663 & 0.9412 & 0.9090 & 0.8773 & \\
Numerical method & 0.9966 & 0.9921 & 0.9825 & 0.9661 & 0.9415 & 0.9181 & 0.8781 & \multirow{2}{*}{25} \\
$\mathrm{t}$ & 18 & 19 & 20 & 21 & 22 & 23 & 24 & 25 \\
\hline MC Simulation & 0.8858 & 0.9025 & 0.9228 & 0.9395 & 0.9466 & 0.9392 & 0.9258 & 0.9142 \\
Numerical method & 0.8778 & 0.9119 & 0.9307 & 0.9591 & 0.9481 & 0.9420 & 0.9095 & 0.8642
\end{tabular}

Table 1: Pointwise availability at time $t$ versus $t$

Table 1 and Figure 6 show the pointwise availability $A_{0}(t)$ calculated using strict Monte Carlo simulation and the pointwise availability calculated by the previous numerical method for different values of $t$. Data for Monte Carlo method have been obtained for 13 equally spaced points ranging from 11 to 23 with 50000 simulations in each point. Both methods mostly coincides for $t$ smaller than $2 \tau=16$ time units. Monte Carlo method however shows a smoother behaviour when compared to the numerical method for larger $t$.

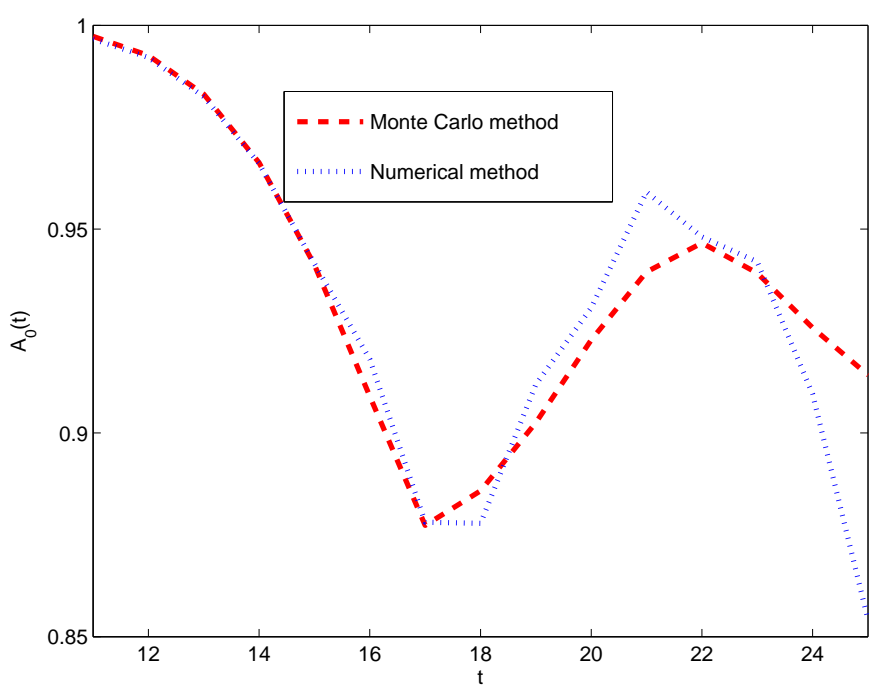

Figure 6: Pointwise availability at time $t$ versus $t$

Taking into account (12) and using the middle point method to approximate integrals, we can estimate the expected cumulated uptime from (17) and (18). For fixed $x$, we consider a grid $\left\{w_{i}, i=1,2, \ldots 15\right\}$ composed of 15 equally spaced points of the interval $[0, t]$ given by

$$
w_{i}=(i-1) \frac{t}{14}, \quad i=1,2, \ldots, 15, \Delta w=w_{i+1}-w_{i} .
$$

We set $w_{i}^{*}$ to be the middle point of this grid, that is, $w_{i}^{*}=w_{i}+\Delta w / 2$, and, from (12), we get an estimation of the expected cumulated uptime as follows

$$
\widehat{m}_{x}(t)=\sum_{j=1}^{15} \widehat{A}_{x}\left(w_{j}^{*}\right) \Delta w,
$$

where $\widehat{A}_{x}$ is given by (17) and (18). 
For different values of $t$, Figure 7 shows the uptime proportion in an interval of time calculated using strict Monte Carlo simulation and the uptime proportion calculated from the numerical method explained above. Data for Monte Carlo method have been obtained for 13 equally spaced points ranging from 9 to 23 with 30000 simulations in each point. For values of $t$ greater than 16, the difference between both methods increases considerably. As in Figure 6, Monte Carlo method shows a smoother behaviour than the numerical method.

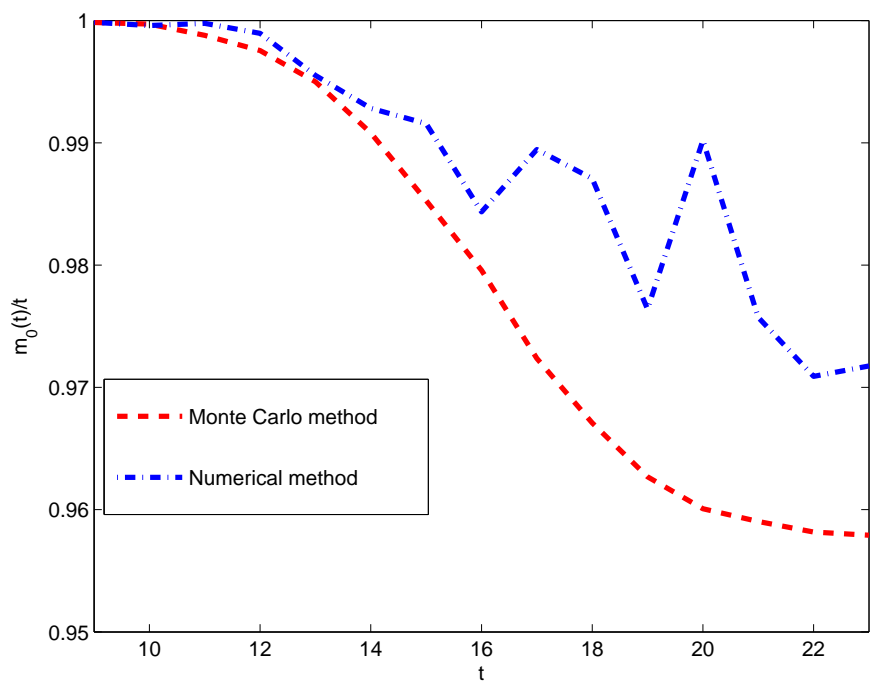

Figure 7: Proportion of the expected cumulated uptime at time $t$ versus $t$

\section{Conclusions}

In this paper, the functioning of a system subject to imperfect maintenance and delayed repair is analyzed. The deterioration of the system is modelled using a gamma process and the functioning of the system is described through a semi-regenerative process with continuous state space. Since the overshoot of the gamma process is taken into account, a complex stochastic model is developed to analyze the transient behaviour of the system. Also, this paper shows a numerical method to obtain the numerical solution of Markov renewal equations fulfilled by some performance measures of the system. This numerical method combines Monte Carlo simulation, interpolation techniques and numerical integration. Up to our knowledge, most of the works that deal with semi-regenerative processes in continuous state space use Monte Carlo simulation to approximate the solutions of the Markov renewal equations. The development of this numerical method hence represents an important contribution of the paper. Its main interest is that is allows for a recursive computation of the performance measures. The kernel is computed once for all at the beginning, as well as the performance measures on $[0, \tau]$, starting from any given initial deterioration level $Y_{0}$. The computation is next made on $(\tau, 2 \tau],(2 \tau, 3 \tau], \ldots$ using the same kernel whatever $Y_{0}$ and the performance measure of interest are. In case several performance measures are required starting from several different possible initial deterioration levels, one could easily think that it should save computation times when compared to strict Monte-Carlo simulations. However, based on the numerical results, it seems that the error made on the kernel and on the computation of the performance measures on $[0, \tau]$ is quickly propagating over the successive time intervals (especially when dealing with a 
unitary performance measure per unit time as in Figure 7). We consequently would suggest to use the numerical method when the point is to compute the performance measures up to a short horizon time (mostly up to $2 \tau$ or $3 \tau$ ) but to use strict Monte-Carlo simulations for larger $t$.

\section{Appendix}

\section{Proof of Theorem 2.}

Given $Y_{0}=x$, we set $S^{x}=S_{1}=\tau+\sigma_{M-x}$. This provides $Y_{S_{1}^{-}}=x+X_{S^{x}}$ and

$$
Y_{S_{1}}=\left\{\begin{aligned}
0 & \text { if } X_{S^{x}}>\min \left(L-x, \frac{M-x}{1-\rho}\right) \\
x+(1-\rho) X_{S^{x}} & \text { if } X_{S^{x}} \leq \min \left(L-x, \frac{M-x}{1-\rho}\right) .
\end{aligned}\right.
$$

For all $\varphi$ measurable and non negative, we hence have:

$$
\mathbb{E}_{x}\left(\varphi\left(S_{1}, Y_{S_{1}^{-}}, Y_{S_{1}}\right)\right)=J_{1}(x)+J_{2}(x)
$$

with

$$
\begin{aligned}
& \left.J_{1}(x)=\mathbb{E}\left[\varphi\left(S^{x}, x+X_{S^{x}}, x+(1-\rho) X_{S^{x}}\right) \times \mathbf{1}_{\left\{X_{S^{x}} \leq \min \right.}\left(L-x, \frac{M-x}{1-\rho}\right)\right\}\right], \\
& J_{2}(x)=\mathbb{E}\left[\varphi\left(S^{x}, x+X_{S^{x}}, 0\right) \times \mathbf{1}_{\left.\left\{X_{S^{x}}>\min \left(L-x, \frac{M-x}{1-\rho}\right)\right\}\right]}\right]
\end{aligned}
$$

Based on Lemma 1, the joint p.d.f. of $\left(S_{x}, X_{S^{x}}\right)$ is $h^{M-x}(\cdot, \cdot)$. We derive:

$$
\begin{array}{r}
J_{1}(x)=\iint_{\mathbb{R}_{+}^{2}} \varphi(s, x+u, x+(1-\rho) u) \mathbf{1}_{\left\{u \leq \min \left(L-x, \frac{M-x}{1-\rho}\right)\right\}} h^{M-x}(s, u) d s d u \\
=\iiint_{\mathbb{R}_{+}^{3}} \varphi(s, y, z) \mathbf{1}_{\{z-x \leq \min ((1-\rho)(L-x), M-x)\}} h^{M-x}\left(s, \frac{z-x}{1-\rho}\right) \mathbf{1}_{\{s>\tau\}} \mathbf{1}_{\{z>x\}} \\
\times \frac{1}{1-\rho} d s \delta_{x+\frac{z-x}{1-\rho}}(d y) d z
\end{array}
$$

and

$$
\begin{aligned}
J_{2}(x) & =\iint_{\mathbb{R}_{+}^{2}} \varphi(s, x+u, 0) \mathbf{1}_{\left\{u>\min \left(L-x, \frac{M-x}{1-\rho}\right)\right\}} h^{M-x}(s, u) \mathbf{1}_{\{s>\tau\}} d s d u \\
& =\iiint_{\mathbb{R}_{+}^{3}} \varphi(s, y, z) \mathbf{1}_{\left\{y-x>\min \left(L-x, \frac{M-x}{1-\rho}\right)\right\}} h^{M-x}(s, y-x) \mathbf{1}_{\{s>\tau\}} d s d y \delta_{0}(d z),
\end{aligned}
$$

which provides the result.

\section{Proof of Corollary 4.}

The sub-kernel $q_{x}^{*}(d s, d z)$ restricted to the operating states is provided by:

$$
q_{x}^{*}(d s, d z)=\int_{0}^{L} Q_{x}(d s, d y, d z)
$$


where the integration is made with respect of $d y$. This provides:

$$
\begin{aligned}
q_{x}^{*}(d s, d z) & =\bar{q}_{x}(s, z) d s\left(\int_{0}^{L} \delta_{x+\frac{z-x}{1-\rho}}(d y)\right) d z \\
& +\mathbf{1}_{\{s>\tau\}} \mathbf{1}_{\left\{x+\min \left(L-x, \frac{M-x}{1-\rho}\right) \leq L\right\}}\left(\int_{x+\min \left(L-x, \frac{M-x}{1-\rho}\right)}^{L} h^{M-x}(s, y-x) d y\right) d s \delta_{0}(d z) .
\end{aligned}
$$

Noticing that $\bar{q}_{x}(s, z)>0$ implies that $z-x \leq(1-\rho)(L-x)$ and consequently $x+\frac{z-x}{1-\rho} \leq L$, we get $\int_{0}^{L} \delta_{x+\frac{z-x}{1-\rho}}(d y)=1$ and the result, noting that

$$
\begin{array}{r}
\mathbf{1}_{\left\{x+\min \left(L-x, \frac{M-x}{1-\rho}\right) \leq L\right\}} \int_{x+\min \left(L-x, \frac{M-x}{1-\rho}\right)}^{L} h^{M-x}(s, y-x) d y \\
=\mathbf{1}_{\left\{x \geq \frac{M-(1-\rho) L}{\rho}\right\}}\left(\int_{\frac{M-x}{1-\rho}}^{L-x} h^{M-x}(s, u) d u\right)
\end{array}
$$

with $u=y-x$.

\section{Proof of Corollary 7.}

The case $i=1$ is provided by (5). As for the recursive formula (11), starting from (6) and taking $t$ such that $i \tau<t \leq(i+1) \tau$, we have:

$$
\begin{aligned}
& A_{x}^{(i+1)}(t) \\
& =G_{x}(t)+\int_{\tau}^{t-(i-1) \tau} \int_{0}^{M} A_{y}(t-s) q_{x}(d s, d y)+\sum_{k=1}^{i-1} \int_{t-k \tau}^{t-(k-1) \tau} \int_{0}^{M} A_{y}(t-s) q_{x}(d s, d y) .
\end{aligned}
$$

Now, as $q_{x}(d s, d y)$ is null on $[0, \tau] \times[0, M]$ (because $S_{1}>\tau$ almost surely), we have

$$
\int_{t-i \tau}^{\tau} \int_{0}^{M} A_{y}(t-s) q_{x}(d s, d y)=0 .
$$

We can hence write:

$$
\int_{\tau}^{t-(i-1) \tau} \int_{0}^{M} A_{y}(t-s) q_{x}(d s, d y)=\int_{t-i \tau}^{t-(i-1) \tau} \int_{0}^{M} A_{y}(t-s) q_{x}(d s, d y)
$$

and

$$
\begin{aligned}
A_{x}^{(i+1)}(t) & =G_{x}(t)+\sum_{k=1}^{i} \int_{t-k \tau}^{t-(k-1) \tau} \int_{0}^{M} A_{y}(t-s) q_{x}(d s, d y) \\
& =G_{x}(t)+\sum_{k=1}^{i} \int_{t-k \tau}^{t-(k-1) \tau} \int_{0}^{M} A_{y}(t-s) \bar{q}_{x}(s, y) d s d y \\
& +\sum_{k=1}^{i} \int_{t-k \tau}^{t-(k-1) \tau} A_{0}(t-s) \stackrel{\circ}{x}_{x}(s) d s .
\end{aligned}
$$


Setting $v_{k}=s-t+k \tau$ in the $k-t h$ term of each sum, we get

$$
\begin{aligned}
A_{x}^{(i+1)}(t) & =G_{x}(t)+\sum_{k=1}^{i} \int_{0}^{\tau} \int_{0}^{M} A_{y}\left(k \tau-v_{k}\right) \bar{q}_{x}\left(t+v_{k}-k \tau, y\right) d v_{k} d y \\
& +\sum_{k=1}^{i} \int_{0}^{\tau} A_{0}\left(k \tau-v_{k}\right) \stackrel{\circ}{q}_{x}\left(t+v_{k}-k \tau\right) d v_{k}
\end{aligned}
$$

and the result, noting that $A_{y}\left(k \tau-v_{k}\right)=A_{y}^{(k)}\left(k \tau-v_{k}\right)$ because $(k-1) \tau<k \tau-v_{k} \leq k \tau$.

\section{Proof of Theorem 10.}

First, we consider the case $\tau \leq t$. We have

$$
\begin{aligned}
I R_{x}(t, t+s) & =\mathbb{P}_{x}\left(Y_{u}<L ; \forall u \in[t, t+s]\right) \\
& =\mathbb{P}_{x}\left(Y_{u}<L ; S_{1}>t+s, \forall u \in[t, t+s]\right)+\mathbb{P}_{x}\left(Y_{u}<L ; t<S_{1}<t+s, \forall u \in[t, t+s]\right) \\
& +\mathbb{P}_{x}\left(Y_{u}<L ; S_{1} \leq t, \forall u \in[t, t+s]\right) .
\end{aligned}
$$

We calculate the three terms of the above equality. The first term is

$$
\begin{aligned}
\mathbb{P}_{x}\left(Y_{u}<L ; S_{1}>t+s, \forall u \in[t, t+s]\right) & =\mathbb{P}\left(X_{t+s}<L-x, \tau+\sigma_{M-x}>t+s\right) \\
& =\mathbb{P}\left(X_{t+s}<L-x, X_{t+s-\tau}<M-x\right) .
\end{aligned}
$$

This term is similar to the first term in (6), proved in [17]. We however recall its computation here, for sake of completeness. Starting again from (20), we first write:

$$
\begin{aligned}
& \mathbb{P}_{x}\left(Y_{u}<L ; S_{1}>t+s, \forall u \in[t, t+s]\right)=\mathbb{P}\left(X_{t+s}-X_{t+s-\tau}<L-x-X_{t+s-\tau}, X_{t+s-\tau}<M-x\right) \\
& =\mathbb{E}\left[\mathbf{1}_{\left\{X_{t+s-\tau}<M-x\right\}} \mathbb{E}\left(\mathbf{1}_{\left\{X_{t+s}-X_{t+s-\tau}<L-x-X_{t+s-\tau}\right.} \mid X_{t+s-\tau}\right)\right] .
\end{aligned}
$$

Based on the independent and homogeneous increments of a gamma process, we have:

$$
\mathbb{E}\left(\mathbf{1}_{\left\{X_{t+s}-X_{t+s-\tau}<L-x-X_{t+s-\tau}\right\}} \mid X_{t+s-\tau}\right)=F_{\tau}\left(L-x-X_{t+s-\tau}\right) .
$$

This provides:

$$
\begin{aligned}
\mathbb{P}_{x}\left(Y_{u}<L ; S_{1}>t+s, \forall u \in[t, t+s]\right) & =\mathbb{E}\left(\mathbf{1}_{\left\{X_{t+s-\tau}<M-x\right\}} F_{\tau}\left(L-x-X_{t+s-\tau}\right)\right) \\
& =\int_{0}^{M-x} f_{t+s-\tau}(u) F_{\tau}(L-x-u) d u \\
& =G_{x}(t+s) .
\end{aligned}
$$

For the second term of (19), we have

$$
\begin{aligned}
& \left.\mathbb{P}_{x}\left(Y_{u}<L ; t<S_{1} \leq t+s, \forall u \in[t, t+s]\right)=\mathbb{E}_{x}\left[\mathbf{1}_{\left\{t<S_{1} \leq t+s\right\}} \mathbf{1}_{\left\{Y_{S_{1}^{-}}<L\right\}}\right\}_{\left\{Y_{u}<L, \forall u \in\left[S_{1}, t+s\right]\right\}}\right] \\
& =\mathbb{E}_{x}\left[\mathbf{1}_{\left\{t<S_{1} \leq t+s\right\}} \mathbf{1}_{\left\{Y_{S_{1}^{-}}<L\right\}} \mathbb{E}\left(\mathbf{1}_{\left\{Y_{u}<L, \forall u \in\left[S_{1}, t+s\right]\right\}} \mid\left(S_{1}, Y_{S_{1}^{-}}, Y_{S_{1}}\right)\right)\right] .
\end{aligned}
$$

Based on the Markov property, we have

$$
\mathbb{E}\left(\mathbf{1}_{\left\{Y_{u}<L, \forall u \in\left[S_{1}, t+s\right]\right\}} \mid\left(S_{1}, Y_{S_{1}^{-}}, Y_{S_{1}}\right)\right)=I R_{Y_{S_{1}}}\left(0, t+s-S_{1}\right)=R_{Y_{S_{1}}}\left(t+s-S_{1}\right) .
$$


We derive:

$$
\begin{aligned}
\mathbb{P}_{x}\left(Y_{u}<L ; t<S_{1} \leq t+s, \forall u \in[t, t+s]\right) & =\mathbb{E}_{x}\left[\mathbf{1}_{\left\{t<S_{1} \leq t+s\right\}} \mathbf{1}_{\left\{Y_{S_{1}^{-}}<L\right\}} R_{Y_{S_{1}}}\left(t+s-S_{1}\right)\right] \\
& =\int_{t}^{t+s} \int_{0}^{M} R_{y}(t+s-u) q_{x}^{*}(d u, d y) .
\end{aligned}
$$

Finally, for the last term of (19), we get

$$
\begin{aligned}
\mathbb{P}_{x}\left(Y_{u}<L ; S_{1}<t, \forall u \in[t, t+s]\right) & =\mathbb{E}_{x}\left[\mathbf{1}_{\left\{S_{1}<t\right\}} \mathbb{E}\left(\mathbf{1}_{\left\{Y_{u}<L, \forall u \in[t, t+s]\right\}} \mid\left(S_{1}, Y_{S_{1}}\right)\right)\right] \\
& =\mathbb{E}_{x}\left[\mathbf{1}_{\left\{S_{1}<t\right\}} I R_{Y_{S_{1}}}\left(t-S_{1}, t+s-S_{1}\right)\right] \\
& =\int_{\tau}^{t} \int_{0}^{M} I R_{y}(t-u, t+s-u) q_{x}(d u, d y),
\end{aligned}
$$

which ends the proof in the case $\tau \leq t$.

Let us now assume $t<\tau<t+s$. We have

$$
\begin{aligned}
I R_{x}(t, s) & =\mathbb{P}_{x}\left(Y_{u}<L, \forall u \in[t, t+s] ; t+s<S_{1}\right)+\mathbb{P}_{x}\left(Y_{u}<L, \forall u \in[t, t+s] ; t+s \geq S_{1}\right) \\
& =G_{x}(t+s)+\mathbb{P}_{x}\left(Y_{S_{1}^{-}}<L ; Y_{u}<L, \forall u \in\left[S_{1}, t+s\right] ; t+s \geq S_{1}\right) \\
& =G_{x}(t+s)+\mathbb{E}_{x}\left[\mathbf{1}_{\left\{t+s \geq S_{1}\right\}} \mathbf{1}_{\left\{Y_{S_{1}^{-}}<L\right\}} \mathbb{E}\left(\mathbf{1}_{\left\{Y_{u}<L, \forall u \in\left[S_{1}, t+s\right]\right\}} \mid\left(S_{1}, Y_{S_{1}^{-}}, Y_{S_{1}}\right)\right)\right] \\
& =G_{x}(t+s)+\mathbb{E}_{x}\left[\mathbf{1}_{\left\{t+s \geq S_{1}\right\}} \mathbf{1}_{\left.Y_{S_{1}^{-}}<L\right\}} R_{Y_{S_{1}}}\left(t+s-S_{1}\right)\right] \\
& =G_{x}(t+s)+\int_{\tau}^{t+s} \int_{0}^{M} R_{y}(t+s-u) q_{x}^{*}(d u, d y)
\end{aligned}
$$

using similar arguments.

\section{Acknowledgments}

For I.T. Castro, this research was supported by Ministerio de Econom'1a y Competitividad, Spain (Project MTM2012-36603-C02-01) and Gobierno de Extremadura, Spain (Project GRU10110).

\section{References}

[1] S. Asmussen. Applied Probability and Queues, Volume 51 of Applications of Mathematics. Springer-Verlag, New York, second edition, 2003.

[2] R.E. Barlow and F. Proschan. Mathematical Theory of Reliability, John Wiley and Sons, 1965.

[3] J. Bertoin. Lévy Processes, Volume 121 of Cambridge Tracts in Mathematics. Cambridge University Press, Cambridge, 1996.

[4] C. Cocozza-Thivent. Processus stochastiques et fiabilité des systémes. Collection Mathématiques et Applications, no. 28, Springer: Berlin Heidelberg New York, 1997 
[5] A. Csenki. An integral equation approach to the interval reliability of systems modelled by finite semi-Markov processes. Reliability Engineering \& System Safety,47(1): 37-45, 1995.

[6] A. Csenki. A new approach to the cumulative operational time for semi-Markov models of repairable systems. Reliability Engineering \& System Safety,54(1): 11-21, 1995.

[7] A. Csenki. Transient Analysis of Semi-Markov Reliability Models - A Tutorial Review with Emphasis on Discrete-Parameter Approaches. In Stochastic Models in Reliability and Maintenance, Osaki S. (Editor). Springer-Verlag Berlin Heidelberg, 219-251, 2002.

[8] P. Do Van, A. Voisin, E. Levrat, B. Iung. A proactive condition-based maintenance strategy with both perfect and imperfect maintenance actions. Reliability Engineering \& System Safety, 133: 22-32, 2015.

[9] D.A. Elkins and M.A. Wortman. On numerical solution of the Markov renewal equation: tight upper and lower kernel bounds. Methodology and Computing in Applied Probability, 3: 239-253, 2001.

[10] S.S. Farahani. The effect of imperfect maintenance on deterioration processes. M.Sc. Thesis, Technical university of Delft, July 2008.

[11] M. Kijima. Some results for repairable systems with general repair. Journal of Applied Probability, 26: 89-102, 1989.

[12] N. Limnios. Reliability measures of semi-Markov systems with general state space. Methodology and Computing in Applied Probability, 14(4): 895-917, 2012.

[13] C. Meier-Hirmer, G. Riboulet, F. Sourget and M. Roussignol. Maintenance optimization for a system with a gamma deterioration process and intervention delay: application to track maintenance. Proceedings of the Institution of Mechanical Engineers, Part O: Journal of Risk and Reliability, 223(3): 189-198, 2009.

[14] S. Mercier and M. Roussignol, Asymptotic failure rate of a Markov deteriorating system with preventive maintenance. Journal of Applied Probability, 40(1): 1-19, 2003.

[15] S. Mercier. Numerical bounds for semi-Markovian quantities and application to reliability. Methodology and Computing in Applied Probability, 10(2): 179-198, 2008.

[16] S. Mercier. Availability in stochastic models. Wiley Enclyclopedia of Operations Research and Management Science, 2010.

[17] S. Mercier and I.T. Castro. On the modelling of imperfect repairs for a continuously monitored gamma wear process through age reduction. Journal of Applied Probability, 50(5): 909-1216, 2013.

[18] R.P. Nicolai, J.B.G. Frenk and R Dekker. Modelling and optimizing imperfect maintenance of coatings on steel structures. Structural Safety, 31(3), 234-244, 2009.

[19] A. Ponchet, M. Fouladirad and A. Grall. Maintenance policy on a finite time span for a gradually deteriorating system with imperfect improvements. Proceedings of the Institution of Mechanical Engineers, Part O: Journal of Risk and Reliability, 225(2): 105-116, 2011. 